\section{Magnetic compass orientation}

SIR - Recent behavioural evidence suggests that the mechanism underlying magnetic compass orientation in newts is light-dependent ${ }^{1,2}$. Among these results are: (1) when newts are orienting magnetically, the direction in which they are moving changes when the animals are exposed to certain specific wavelengths of light ${ }^{1}$; and (2) newts orient magnetically under full-spectrum light but not in the absence of visible light ${ }^{2}$. Such results are consistent with models proposing that magnetoreception involves a modulation of the response of retinal photoreceptors to light, and therefore cannot occur in darkness.

Light is not necessary, however, for magnetic compass orientation in all vertebrates. Recent experiments with loggerhead sea turtle hatchlings have demonstrated that tethered turtles swimming in complete darkness can orient to the Earth's magnetic field ${ }^{3}$. I have recently repeated this finding in both loggerhead hatchlings and in a second species of marine turtle, the leatherback Dermochelys coriacea (unpublished data). Representatives of two other vertebrate classes (fish ${ }^{4}$ and mammals ${ }^{5}$ ) also probably orient magnetically in darkness, as do several invertebrates $^{6,7}$. These results suggest that light-independent magnetic compasses are phylogenetically widespread.

The light-independent compass of sea turtles and the light-dependent compass of newts may or may not rely on the same transduction process. The turtle compass $^{8}$, however, resembles the magnetic compass of shorewards-orienting newts ${ }^{9}$ in that both compasses are axial and based on field-line inclination rather than on field polarity. Thus, light-dependence is apparently not a universal feature of vertebrate magnetic compasses, of invertebrate magnetic compasses, or of inclination compasses.

Magnetoreception in darkness may still occur in photoreceptors if the transduction process relies on magnetic fielddependent biochemical reactions ${ }^{10}$ that are independent of light. In principle, such reactions could occur anywhere in

1. Phillips, J. B. \& Borland, S. C. Nature $359,142-144$ (1992).

2. Phillips, J. B. \& Borland, S. C. Anim. Behav. 44, 796-797 (1992)

3. Lohmann, K. J. J. exp. Biol. 155, $37-49$ (1991).

4. Quinn, T. P. J. comp. Physiol. A137, 243-248 (1980)

5. Burda, H., Marhold, S., Westenberger, T., Wiltschko, R. \& Wiltschko, W. Experientia 46, 528-530 (1990)

6. Arendse, M. C. Nature 274, 358-362 (1978).

7. Lohmann, K. J. \& Willows, A. O. D. Science 235 , 331-334 (1987)

8. Light, P., Salmon, M. \& Lohmann, K. J. J. exp. Biol. (in the press).

9. Phillips, J. B. Science 233, 765-767 (1986)

10. Schulten, K. \& Windemuth, A. in Biophysical Effects of Steady Magnetic Fields (eds Maret, G., Boccara, N. \& Kiepenheuer, J.) 99-106 (Springer, Berlin, 1986). the body, but the photoreceptors are an appealing location because the retina provides an ordered array of receptor molecules. At present, however, there is no direct evidence for such a lightindependent biochemical transduction mechanism, either in photoreceptors or elsewhere. Further research is required compasses capable of functioning in darkness rely on different receptors from the apparently light-dependent magnetic compass of newts, or whether all of these compasses in fact share similar underlying receptor mechanisms.

\section{Kenneth J. Lohmann}

Department of Biology,

University of North Carolina,

Chapel Hill,

North Carolina 27599-3280, USA

\section{Electrochemical mercury detection}

SIR - Concern about the environmental dangers of mercury pollution has led to remarkable efforts towards developing analytical methods for this metal. To determine the environmental levels of mercury, which can be of the order of a few parts per trillion $\left(10^{12}\right)$ (p.p.t.), highly sensitive and selective methods must be used. However, most approaches require a preconcentration or a pretreatment step owing to their low sensitivity or selectivity ${ }^{1,2}$. We report here on the application of a glassy carbon electrode (GCE) spin-coated with 4,7,13,16,21,24-hexaoxa-1,10-diazabicyclo[8.8.8] hexacosane (Kryptofix-222) for very selective determination of ultra low levels $\left(<10^{-12} \mathrm{M}\right)$ of mercury. to determine whether magnetic
The figure shows the voltammetric analysis of sea water (Mediterranean Sea, Israel) obtained with a modified electrode without further treatment. The anodic stripping Osteryoung square wave voltammograms (OSWV) reveal a clear anodic peak at $0.1 \mathrm{~V}$, associated with the oxidation of predeposited mercury $(5 \mathrm{~min}$ deposition time at $-0.5 \mathrm{~V})$. Electrodes were electrochemically regenerated after each experiment (soaking in solution for $1 \mathrm{~min}$ at $0.3 \mathrm{~V}$ ) and, as a result, could be applied for numerous experiments. The concentration of mercury in sea water was estimated as $(1.01 \pm 0.03) \times 10^{-11} \mathrm{M}(2$ p.p.t. $)$ using a standard addition method.

Optimizing the various parameters that affect the sensitivity of mercury determination, such as potential and time of deposition, resulted in a linear dependence between the stripping peak current and concentration of $\mathrm{Hg}$ (II) in the range of $1.5 \times 10^{-12}$ to $1.2 \times 10^{-11}$ M. The detection limit is less than $10^{-12}$ M ( 0.2 p.p.t. $)$ with a relative standard deviation of $3.3 \%$.

To confirm our results as well as to verify the application of the developed method to the analysis of natural waters, simultaneous analyses have been accomplished by our method and by cold vapour flameless atomic absorption. Excellent agreement between the results obtained from both methods has been found. For example, the concentrations of mercury in two samples were $0.75 \pm$ 0.06 and $82.2 \pm 0.1$ p.p.b. as compared with 0.90 and 89.5 p.p.b., respectively, obtained by flameless atomic absorption. These samples had to be diluted by 450 and 45,000 times, respectively, before voltammetric analysis could be pursued.

The fact that the results obtained from the analysis of natural samples by our method were in good agreement with another analytical method suggests that

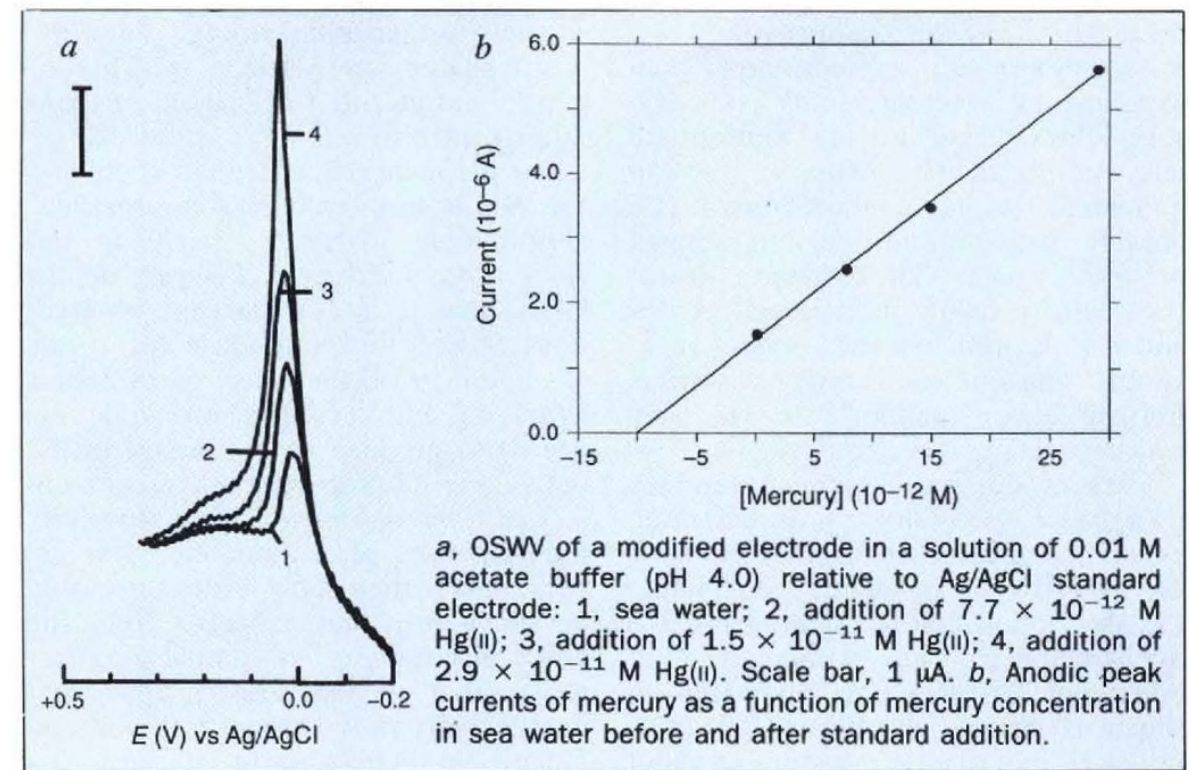

